\title{
Bioinformatics Analysis and Characteristics of the giant panda Interferon-alpha
}

\author{
YueYi \\ Animal Biotechnology Center College of Veterinary Medicine of Sichuan Agricultural University, Ya'an, \\ Sichuan,625014, China \\ E-mail:yiyue19870301@126.com \\ Zhiwen Xu \\ Animal Biotechnology Center College of Veterinary Medicine of Sichuan Agricultural University, Ya'an, \\ Sichuan, 625014, China
}

\begin{abstract}
In this report, the amino acid sequence of giant panda interferon- $\alpha$ (gpIFN- $\alpha$ ) was determined and compared with 15 corresponding IFN- $\alpha$ sequences. Phylogenetic analysis showed that the 15 interferons fell into two large groups. The giant panda and ferret branched and were most closely related to fox and $\mathrm{dog}$ and evolved into a distinct phylogenetic lineage from that of eukaryotic mammalians which evolved into another lineage. After analyzing the encoded amino acid sequence of the gpIFN-a using bioinformatics, the results revealed that in the full amino acid sequence, there were no transmembrane domain, one N-glycosylation sites, eight O-glycosylation sites and nine antigenic determinants. Secondary structure analyzed showed that the Alpha helix, Extended strand, Beta turn and Random coil each occupied 60.37\%(99aa), $4.88 \%(8 a a), 9.76 \%, 25 \%(41 a a)$ respectively. In conclusion, our results will give the opportunity to investigate more in detail function study in giant panda and add to studies on the evolution of the IFN system in vertebrates and avian more generally.
\end{abstract}

Index Terms-Ailuropoda melanoleuca, interferon alpha, clone, sequence analysis, Structure analysis

\section{I . INTRODUCTION}

Interferons (IFNs) are a multi-gene family of inducible cytokines which have a major role in immune defense

Corresponding author: Zhiwen Xu;

E-mail:abtcxzw@126.com against virus infections but are also recognized for their antiproliferative and immunomodulatory activities $[1,2]$. IFNs are produced in numerous cell types including lymphocytes ( $\mathrm{T}$ and $\mathrm{B}$ cells), macrophages, fibroblasts, blood vessel endothelial cells and osteogenetic cells, and are known to be important elements in antivirus reply [3]. They were classified as Type I IFNs (virus-infected IFNs), Type II IFNs (immune IFNs) and type III [4]. The alphainterferons belonged to type I interferons, are mainly produced by virus-infected peripheral blood leukocytes, lymphobl- astoid and myeloblastoid cell lines[5]. It is considered to be a choice drug to treat virus disease and tumour in clinics.

The giant panda (Ailuropoda melanoleuca) is a much loved animal all over the world and is considered a symbol of China[6], However, it is also one of the world's most endangered species, as well as a flagship species for conservation. The latest molecular censusing research used fecal samples and nine microsatellite loci shows that the estimated number of wild pandas is 1596 , and the number of captive pandas is about 161. In recent years, strenuous efforts have been made to protect this animal and considerable knowledge of its physiology, biochemistry, genetic diversity and ecology has been onrd gained, but death of disease are one of the major problems facing giant panda health. The research on the diseases of giant panda revealed that there were more than 40 kinds of diseases do exist. They are mainly viruses, bacteria and parasite. Therefore, studies of cytokine genes of the giant panda become important and 
necessary to facilitate the use of these cytokines in the immunotherapy of infectious diseases of the giant panda.

During the past decade, a variety of interferon genes have been cloned and sequenced from a number of species, including human, canine, porcine, chicken. And many studies had been performed on them. nonetheless, research on the giant panda Interferon-alpha is still rare. Although the gene has been reported since 2007 [7], very few information about the structures and functions of giant panda IFN- $\alpha$ has been published. In order to understand the bioinformatics and characteristics of the gpIFN- $\alpha$ at the molecular level and to extend the

\section{II . MATERIALS AND METHODS}

\section{A.Culture of peripheral blood lymphocytes}

Adult giant pandas were phlebotomized at the Ya'an Research Base of Giant Panda Breeding under required procedures. Peripheral blood lymphocytes were separated by gradient centrifugation using Lymphocyte Separation Medium (TBD, Tianjin, China), and the Cells $\left(5 \times 10^{6}\right.$ cells $\left./ \mathrm{ml}\right)$ were then resuspended and cultured in RPMI-1640 medium (Gibco, Grand Island,NY, USA) supplemented with $10 \%$ fetal calf serum, $100 \mathrm{U} / \mathrm{ml}$ penicillin, $100 \mathrm{mg} / \mathrm{ml}$ streptomycin. Lymphocytes were stimulated with concanavalin $\mathrm{A}$ at a final concentration of $15 \mathrm{mg} / \mathrm{ml}$ and incubated for $16 \mathrm{~h}$ at $37^{\circ} \mathrm{C}$ in a humidified incubator with $5 \% \mathrm{CO} 2$. After $16 \mathrm{~h}$, the stimulated cells were washed twice with Hanks'balanced salt solution and collected by centrifugation.

\section{B. RNA extraction}

Total RNAs from the harvested cells were isolated using TRIzol reagent (TaKaRa, DaLian, Japan) according to the manufacturer's instruction. RNA quality was evaluated on $1.0 \%$ agarose gels containing ethidium bromide $(10 \mathrm{mg} / \mathrm{mL})$. RNA samples were used subsequently for RT-PCR or stored at $-70^{\circ} \mathrm{C}$.

\section{RT-PCR amplification of the IFN-a gene}

Synthesis of the first strand of complementary DNA (cDNA) was performed using an RNA polymerase chain reaction (PCR) kit (TaKaRa, Dalian, Japan). The cDNA was synthesized at $37^{\circ} \mathrm{C}$ from RNA by using oligo-dT (Promega, Madison, WI,USA). The following IFN-a specific primers were used in the amplification: IFN-a applications of recombinant giant panda IFN- $\alpha$, we are trying to analyze and report some information about the structure and function of its encoded protein. Furthermore, to illustrate its evolutionary relationships among the eukaryotic mammalians, a phylogenetic tree was constructed. Our results will give the opportunity to investigate in detail on the evolution of the IFN system in avian and mammals more generally, and may provide some insights for further research the function and biological activities about the $g p$ IFN- $\alpha$.

forward, 5'-GGATCCTGTGACCTGCCTCAGAACCA TGGCCTG-3' containing the BamHI site, and reverse, 5'-GTCGACTCATTTCTCGCTCCTTAGTCTTTCTTG3 'containing the SalI site. The PCR was carried out in a $20 \mu \mathrm{L}$ total volume reaction mixture that consisted of $10 \mu \mathrm{L} 2 \times$ PCR Mixture (TaKaRa, Dalian, Japan), $0.5 \mu \mathrm{L}$ each primer $(25 \mathrm{mM}), 1.0 \mu \mathrm{L}$ template cDNA (about 300 $\mathrm{ng}$ ) and $8 \mu \mathrm{L}$ ddH2O. The conditions of PCR were: $95^{\circ} \mathrm{C}$ for $5 \mathrm{~min}, 35$ repeated cycles of $95^{\circ} \mathrm{C}$ for $30 \mathrm{~s}, 58^{\circ} \mathrm{C}$ for 40 $\mathrm{s}$ and $72^{\circ} \mathrm{C}$ for $45 \mathrm{~s}$, and then a final extension at $72^{\circ} \mathrm{C}$ for $10 \mathrm{~min}$. The products of these PCR were electrophoresed on $1 \%$ agarose gelscontaining ethidium bromide and visualized under an ultraviolet light.

\section{Cloning and Sequencing of the IFN-a Gene}

The PCR products were purified by using a TIANprep Mini Plasmid Kit (TianGen) according to the manufacturer's instructions. The purified PCR products were cloned into pMD19-T vector (TaKaRa), and then transformed into Escherichia coli (E.coli) DH5 $\alpha$ competent cell, After that, the positive recombinant clone was selected by the Amp/IPTG/X-Gal agar plate. Plasmid DNA was identified by bacterial colony PCR with a forementioned conditions, and digested with restriction enzymes BamHI and SalI (TaKaRa, Dalian, Japan). Then it had to be fractionated in $1 \%$ agarose gels. Sequencing reactions was also performed by TaKaRa.

\section{E. Analyzing nucleotide sequence of IFN-a the gene by bioinformatics}

We incorporated IFN- $\alpha$ sequences described for giant panda as well as 14 other mammalian and avian species from NCBI, and their GenBank accession numbers are listed in Table 1. Multiple sequence alignments of 
deduced amino acid sequences of these 15 interferons were performed by using ClustalW multiple alignments. Phylogenetic trees were constructed using the neighbor -joining method in MEGA version 4.0. To estimate the transmembrane domain of the $g p$ IFN- $\alpha$, the amino acid sequence was analyzed by using online prediction tools. Prediction of the transmembrane segment were analyzed es/NetNGlyc/), NetPhos2.0 (http://www.cbs.dtu.dk/ services/NetPhos/), and BepiPred 1.0 Server (http://www.cbs. dtu.dk/services/BepiPred/), respectively. The structure of Intron and exon was analyzed by using Genscan (http://genes.mit.edu/GENSCAN.html). Secondary structure was predicted by using SOPM method on web site (http://npsa-pbil.ibcp.fr/cgi-bin/npsaautomat.pl?page=/ with TMHMM (http://genome.cbs.dtu.dk/services/TMH -MM/). Using the DNAStar 7.0 and on-line predicted tools to analyze the structural properties of the gpIFN- $\alpha$ amino acids sequence. The glycosylation sites, phosphorylation site and epitope were analyzed using the programs of NetNGlyc1.0(http://www.cbs.dtu.dk/servic-

-NPSA/-npsa_server.ht). The similar three dimensional structure was obtained online by Swiss-Model server (http:// swiss -model. expasy.org) and was observed with RasMol 2.7 software. Rare condons content analysis of the $g p$ IFN- $\alpha$.gene by using codon usage data base on line(http://nihserver.mbi.ucla.edu/RACC/).

\begin{tabular}{|c|c|c|c|c|}
\hline Species & GenBank Accession No. & Animals' IFN- $\alpha$ & Natural animal & Length (bp) \\
\hline & AY323972 & Bubalus bubalis IFN- $\alpha$ & Bubalus bubalis & 498 \\
\hline & DQ520882 & Canis familiaris IFN- $\alpha$ & Canis familiaris & 495 \\
\hline & FJ959074 & Capra hircus IFN- $\alpha$ & Capra hircus & 501 \\
\hline & NM_001099441 & Equus caballus IFN- $\alpha$ & Equus caballus & 486 \\
\hline & NM_001009851 & Felis catus IFN- $\alpha$ & Felis catus & 516 \\
\hline & NM_001194384 & Macaca mulatta IFN- $\alpha$ & Macaca mulatta & 501 \\
\hline \multirow[t]{6}{*}{ Mammal } & EU863618 & Mustela putorius furo IFN- $\alpha$ & Mustela putorius furo & 495 \\
\hline & HM636502 & Pteropus vampyrus IFN- $\alpha$ & Pteropus vampyrus & 540 \\
\hline & AY331298 & Sus scrofa IFN- $\alpha$ & Sus scrofa & 501 \\
\hline & EF990625 & Vulpes vulpes IFN- $\alpha$ & Vulpes vulpes & 540 \\
\hline & ВC074928 & Homo sapiens IFN- $\alpha$ & Homo sapiens & 501 \\
\hline & HQ378189 & grint panda IFN- $\alpha$ & grint panda & 495 \\
\hline \multirow[t]{3}{*}{ Birds } & X84764 & duck IFN- $\alpha$ & duck & 492 \\
\hline & AB021154 & Gallus gallus IFN- $\alpha$ & Gallus gallus & 492 \\
\hline & EU022750 & Shitou Goose IFN- $\alpha$ & Shitou Goose & 492 \\
\hline
\end{tabular}

\section{RESULTS}

\section{A. Analysis the amino acids sequences of gpIFN- $a$}

The $g p$ IFN- $\alpha$ gene was composed of 495 nucleotides and was translated into a putative peptide of 164 amino acid residues(Fig. 1). Also it has been submitted to GenBank and the accession number HQ378189 was assigned for the IFN-a of the giant panda. Through on-line (http://www.expasy.org/tools/protparam.html) analysis, it had a putative molecular mass of $18.52 \mathrm{kDa}$ and a predicted isoelectric point (PI) of 6.10. In addition, the results showed that the formular of the $g p \mathrm{IFN}-\alpha$ gene was $\mathrm{C}_{818} \mathrm{H}_{1281} \mathrm{~N}_{225} \mathrm{O}_{246} \mathrm{~S}_{10}$, the total number of atoms was 2580 , the instability index was 49.27 , the aliphatic index was 79.37, and the grand average of hydropathicity (GRAVY) was 0.809. The total number of negative charged residues $(\mathrm{Asp}+\mathrm{Glu})$ and positive charged residues (Arg + Lys) was 18 and 16, respectively. The estimated half-life of gpIFN- $\alpha$ was: 7.2 hours (mammalian reticulocytes, in vitro), $>20$ hours (yeast, in vivo), $>10$ hours (Escherichia coli, in vivo). Through the analysis of DNAStar7.0, the polypeptide encoded by the gpIFN- $\alpha$ was composed of 58 hydrophobic amino acids, 51 hydrophilic amino acids, 16 basic amino acids and 18 
acidic amino acids. Furthermore, through the prediction of GENESCAN on line, the consequence indicated that there was no intros presenting in the sequence.

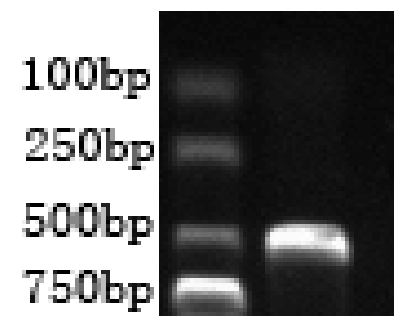

Figure 1. RT-PCR of giant panda IFN-a. The expected about 500bp

fragment of giant panda IFN-a cDNA was amplified.

\section{B. Functional sites analysis}

By using different online web servers, we gained more informations about the $g p \mathrm{IFN}-\alpha$. Such as follows: Firstly, the mature amino acid of $g p \mathrm{IFN}-\alpha$ had one potential $\mathrm{N}$-glycosylation sites and eight potential O-glycosylation sites. The potential $\mathrm{N}$-glycosylation sites located at 78aa, and the biggest potential value was 0.6653 . The O-glycosylation sites located at 70aa, 73aa, 74aa, 109aa, 141aa, 147aa, 148aa, and 162aa respectively. These results revealed that like other mammalian type I IFNs, the gpIFN- $\alpha$ was also a glycoprotein. Secondly, the phosphorylation sites were predicted through on-line analysis software NetPhos2.0 (http://www.cbs.dtu.dk/ services/NetPhos/). The result was shown in fig. 3. When taken the threshold as 0.5, the mature amino acid sequence had a total of eleven potential phosphorylation sites, including six serine sites, four threonine site and one tyrosine site. thirdly, one cAMP-and cGMPdependent protein kinase phosphorylation site (at aa residue 22), two Protein kinase $\mathrm{C}$ phosphorylation sites (at aa residues 141,162 ) and three casein kinase II phosphorylation site (at aa residue $27,80,94$ ) can be clearly identified. And also contain a signature of interferon alpha, beta and delta family (at aa residue 121). Fourthly, there were six cysteines residue respectively locate at 1, 29, 69, 87, 100 and 145 amino acids. At last there were twenty-one protein-protein interaction sites that were likely to be hot spots for protein interaction. 


\begin{tabular}{|c|c|c|c|c|c|c|c|c|c|c|c|c|c|c|c|c|c|c|c|c|}
\hline & 1 & 2 & 3 & 4 & 5 & 6 & 7 & 8 & 9 & 10 & 11 & 12 & 13 & 14 & 15 & 16 & 17 & 18 & 19 & 20 \\
\hline N1 & tgt & gac & ctg & cct & cag & aac & cat & ggc & ctg & $\mathrm{ttt}$ & $\mathrm{gcc}$ & tgg & agg & $g c c$ & $\mathrm{ttg}$ & acg & ctc & ctg & gga & caa \\
\hline \multirow[t]{2}{*}{ P1 } & C & D & $\mathrm{L}$ & $P$ & $Q$ & $\mathrm{~N}$ & $\mathrm{H}$ & G & L & F & A & W & $\mathrm{R}$ & $\mathrm{A}$ & L & $T$ & L & L & G & $Q$ \\
\hline & 21 & 22 & 23 & 24 & 25 & 26 & 27 & 28 & 29 & 30 & 31 & 32 & 33 & 34 & 35 & 36 & 37 & 38 & 39 & 40 \\
\hline N61 & atg & aag & aga & atg & tct & get & agc & tct & tgt & gac & ggg & $\operatorname{tac}$ & $\mathrm{aca}$ & cat & gac & $\mathrm{ttt}$ & $\mathrm{gcc}$ & $\mathrm{ttc}$ & $\operatorname{ccc}$ & aag \\
\hline \multirow[t]{2}{*}{ P21 } & $\mathbf{M}$ & $\mathrm{K}$ & R & M & $\mathrm{S}$ & A & $\mathrm{s}$ & $\mathrm{S}$ & C & D & G & $\mathrm{Y}$ & $\mathrm{T}$ & $\mathrm{H}$ & D & F & A & F & $\mathbf{P}$ & $\mathrm{K}$ \\
\hline & 41 & 42 & 43 & 44 & 45 & 46 & 47 & 48 & 49 & 50 & 51 & 52 & 53 & 54 & 55 & 56 & 57 & 58 & 59 & 60 \\
\hline N121 & gag & gtg & gtt & gat & ggc & aag & cag & $\mathrm{ttg}$ & cag & aag & get & caa & $\mathrm{gcc}$ & ctc & tcg & gtc & gtg & cat & gtg & atg \\
\hline \multirow[t]{2}{*}{ P41 } & $E$ & $\mathrm{~V}$ & V & D & G & $\mathrm{K}$ & $Q$ & $\mathrm{~L}$ & $Q$ & $\mathrm{~K}$ & A & $Q$ & A & $\mathrm{L}$ & $\mathrm{s}$ & V & $\mathrm{V}$ & $\mathrm{H}$ & $\mathrm{V}$ & $\mathrm{M}$ \\
\hline & 61 & 62 & 63 & 64 & 65 & 66 & 67 & 68 & 69 & 70 & 71 & 72 & 73 & 74 & 75 & 76 & 77 & 78 & 79 & 80 \\
\hline N181 & gac & cag & aag & atc & $\mathrm{ttc}$ & $\mathrm{cac}$ & gtc & $\mathrm{ttc}$ & $\operatorname{tgc}$ & aca & gag & $\operatorname{ccc}$ & tca & tct & get & get & $\mathrm{ttg}$ & aac & agg & acc \\
\hline \multirow[t]{2}{*}{ P61 } & D & $Q$ & $\mathrm{~K}$ & I & F & $\mathrm{H}$ & $\mathrm{V}$ & $F$ & C & $\mathrm{T}$ & $\mathrm{E}$ & $P$ & $\mathrm{~S}$ & $\mathrm{~S}$ & A & A & w & $\mathrm{N}$ & $\mathrm{R}$ & $T$ \\
\hline & 81 & 82 & 83 & 84 & 85 & 86 & 87 & 88 & 89 & 90 & 91 & 92 & 93 & 94 & 95 & 96 & 97 & 98 & 99 & 100 \\
\hline N241 & ctc & cta & gag & gaa & $\mathrm{ttc}$ & $\operatorname{tgc}$ & tcg & gga & $\mathrm{ctt}$ & tct & gag & cag & ctg & agc & gtc & ctg & gaa & $\mathrm{gcc}$ & $\operatorname{tgc}$ & $\operatorname{ccc}$ \\
\hline \multirow[t]{2}{*}{ P81 } & L & L & E & $\mathrm{E}$ & $F$ & C & $\mathrm{s}$ & G & L & $\mathrm{S}$ & $\mathrm{E}$ & $Q$ & $\mathrm{~L}$ & s & V & L & E & A & C & $P$ \\
\hline & 101 & 102 & 103 & 104 & 105 & 106 & 107 & 108 & 109 & 110 & 111 & 112 & 113 & 114 & 115 & 116 & 117 & 118 & 119 & 120 \\
\hline N301 & atg & cag & $\mathrm{gcg}$ & $\mathrm{gcg}$ & gga & gtg & gga & gag & act & $\operatorname{ccc}$ & gtc & agg & aat & gtg & gac & tec & atc & $\mathrm{ctg}$ & agg & aac \\
\hline \multirow[t]{2}{*}{ P101 } & $\mathbf{M}$ & $\mathrm{Q}$ & A & A & G & $\mathrm{V}$ & G & $\mathrm{E}$ & $\mathrm{T}$ & $\mathbf{P}$ & V & $\mathrm{R}$ & $\mathrm{N}$ & $\mathrm{V}$ & D & $\mathrm{S}$ & I & $\mathrm{L}$ & $\mathrm{R}$ & $\mathrm{N}$ \\
\hline & 121 & 122 & 123 & 124 & 125 & 126 & 127 & 128 & 129 & 130 & 131 & 132 & 133 & 134 & 135 & 136 & 137 & 138 & 139 & 140 \\
\hline N361 & tac & $\mathrm{ttc}$ & caa & aga & atc & tcc & ctc & tac & ctg & caa & gag & aag & caa & tac & agc & cct & tgt & gcc & tgg & gag \\
\hline \multirow[t]{2}{*}{$\mathrm{P} 121$} & $Y$ & F & $Q$ & R & I & S & I & $\mathrm{Y}$ & L & $Q$ & E & $\mathrm{K}$ & Q & $\mathrm{Y}$ & S & P & C & A & W & E \\
\hline & 141 & 142 & 143 & 144 & 145 & 146 & 147 & 148 & 149 & 150 & 151 & 152 & 153 & 154 & 155 & 156 & 157 & 158 & 159 & 160 \\
\hline $\mathrm{N} 421$ & act & gtc & aga & gca & gaa & atc & acg & agc & tcc & $\mathrm{ttg}$ & $\mathrm{ttt}$ & tca & tca & acg & atc & $\mathrm{ttg}$ & caa & gaa & aga & cta \\
\hline \multirow[t]{2}{*}{ P141 } & $\mathrm{T}$ & $\mathrm{V}$ & R & A & $\mathrm{E}$ & I & $T$ & $\mathrm{~S}$ & $\mathrm{~S}$ & L & F & $\mathrm{S}$ & $\mathrm{S}$ & $T$ & I & L & $Q$ & E & $\mathrm{R}$ & L \\
\hline & 161 & 162 & 163 & 164 & 165 & & & & & & & & & & & & & & & \\
\hline N481 & agg & agc & gaf & aaa & tga & & & & & & & & & & & & & & & \\
\hline P161 & $\mathrm{R}$ & $\mathrm{s}$ & E & $\mathrm{K}$ & $*$ & & & & & & & & & & & & & & & \\
\hline & & & & & & & & & & & & & & & & & & & & \\
\hline
\end{tabular}

Figure 2. Nucleotide sequence of gpIFN- $\alpha$ gene and its deduced amino acid sequence. In the amino acid sequence, there are some repetitional functional sites, we use different colors to distinguish them. the boxed letter highlighted in blue indicates the putative N-glycosylation site; phosphorylation sites are identified by the letters highlighted in purple; N-glycosylation site is identified by the letters highlighted in pink; cAMP-and cGMP-dependent protein kinase phosphorylation site is identified by the letters highlighted in orange; Protein kinase C phosphorylation sites are identified by the letters highlighted in brown; casein kinase II phosphorylation site is identified by the letters highlighted in gray; cysteines residue marked with yellow protein-protein interaction sites are marked with reseda; the interferon alpha, beta and delta family signature is identified by the letters highlighted in green; and also the asterisk indicates the stop codon.

\section{Transmembrane domain and antigenic Determinants of the gpIFN- $\alpha$ protein}

The prediction of transmembrane domain revealed that the $g p \mathrm{IFN}-\alpha$ amino acid had non-transmembrane domain (Fig. 3). Through the prediction of online web servers, we found that $g p \mathrm{IFN}-\alpha$ amino acid sequence contained nine antigenic determinants and was mainly positioned at 1-4aa, 26-33aa, 37aa, 41-49aa, 71-77aa, 101-113aa, 133-139aa, 161-162aa, 164aa (fig. 4).

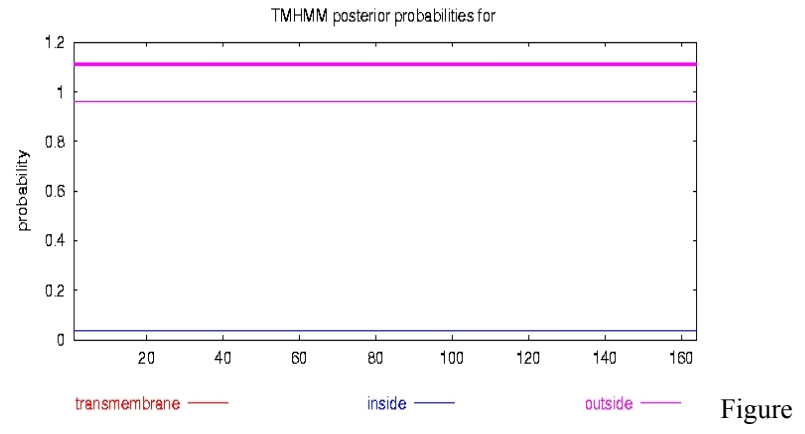

3. The prediction of transmembrane segments. 


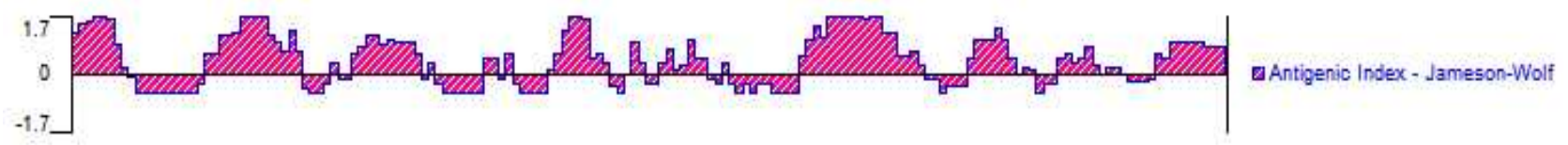

Figure 4. The predicted results of the antigenic determinants. It was performed with DNAStar 7.0.

\section{The prediction of Secondary structure and tertiary} structure of IFN- $a$

The prediction of $g p$ IFN-a's secondary structure was shown in Figure 5(A) and Figure 5(B). The result indicated that the alpha helix $(\mathrm{H})$, extended strand $(\mathrm{E})$, Beta turn and random coil ( $\mathrm{C}$ ) each occupied 60.37\%(99aa), 4.88\%(8aa), 9.76\% (16aa), 25\%(41aa) respectively. The alpha helix of the protein have higher chemical bonding energy, which can firmly maintain proteinic higher structure, and mainly situated in aa 8-26, 40-56, 74-98, 114-130, 139-162. And the rest components are mainly at aa 1-7, 27-39, 44-49, 61-73, 99-113 and 131-138.

The similar three dimensional structure of the IFN-a by Swiss-Model automobile homology modeling database is showed in Fig. 6. The templet is 1itfA with 54.82\% identity. Both of the two pictures were displayed by RasMol 2.7 software with Ribbons mode, the left picture with group colour pattern while the right picture with structure colour pattern.

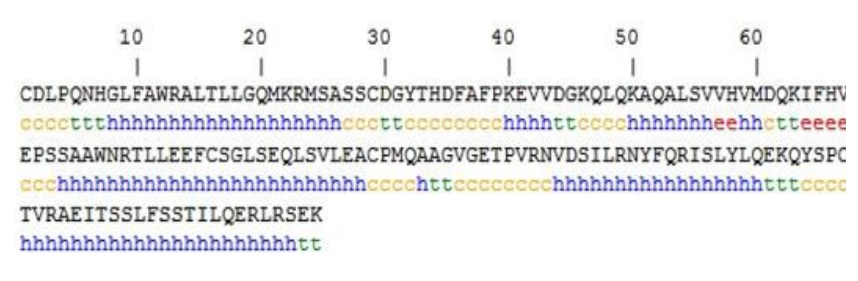

Figure 5(A). The secondary structure of IFN-a is predicted by SOPM method with the online web server (http://npsapbil.ibcp.fr/cgi-bin/npsa_automat.pl?p-age=/NPSA/npsa_ server.html), h represents alpha helix, e represents extended strand, c represents random coil, and t represents beta turn.

\section{E. Similarity and Phylogenetic analysis}

Similarity comparison of gpIFN- $\alpha$ amino acid sequence with those of 14 other mammalian and avian

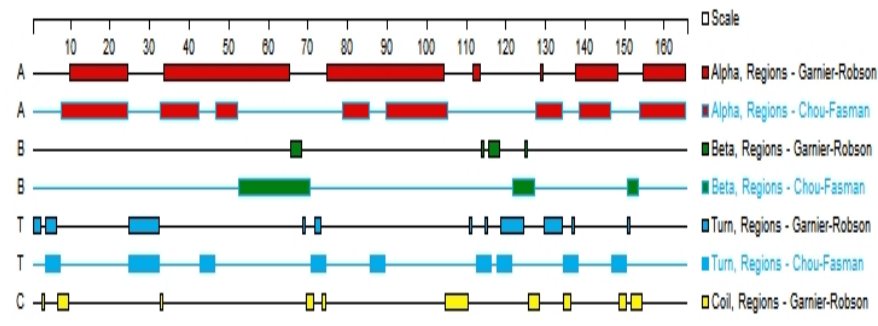

Figure 5(B). The $g p$ IFN- $\alpha$ secondary structure is predicted with DNAStar 7.0

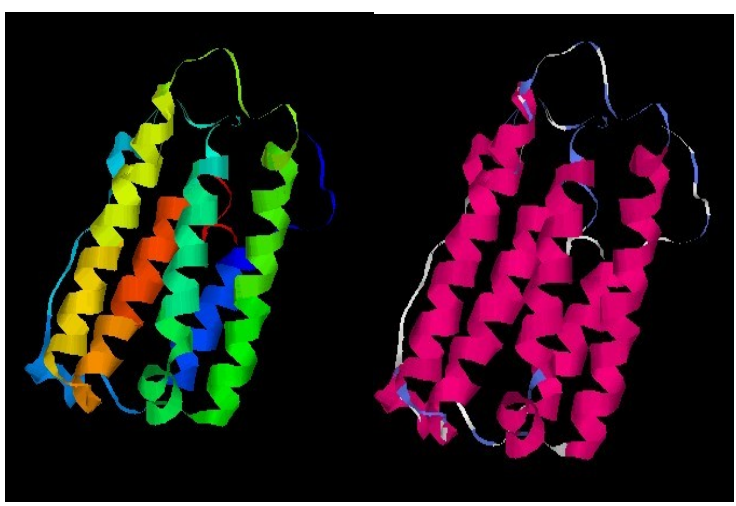

Figure 6. The 3D model of IFN-a. The left with group colour pattern: according to their location, that amino acids are colored from blue, green, yellow, orange to red, with the $\mathrm{N}^{\prime}$ terminal as blue, the $\mathrm{C}^{\prime}$ terminal as red.The right with structure colour pattern: according to their secondary structure, that amino acids are colored with the alpha helix as scarlet, the turn as light blue, other as white.

types of human, ferret, dog, cat, fox, chicken and duck IFN-a were approximately $58.2 \%, 73.9 \%, 70.3 \%, 70.9 \%$, $70.3 \%, 26.2 \%, 21.3 \%$, respectively (Fig. 7). This revealed the big similarity between the giant panda and ferret. This may provide some clues to explore the enigmatic relationship. 

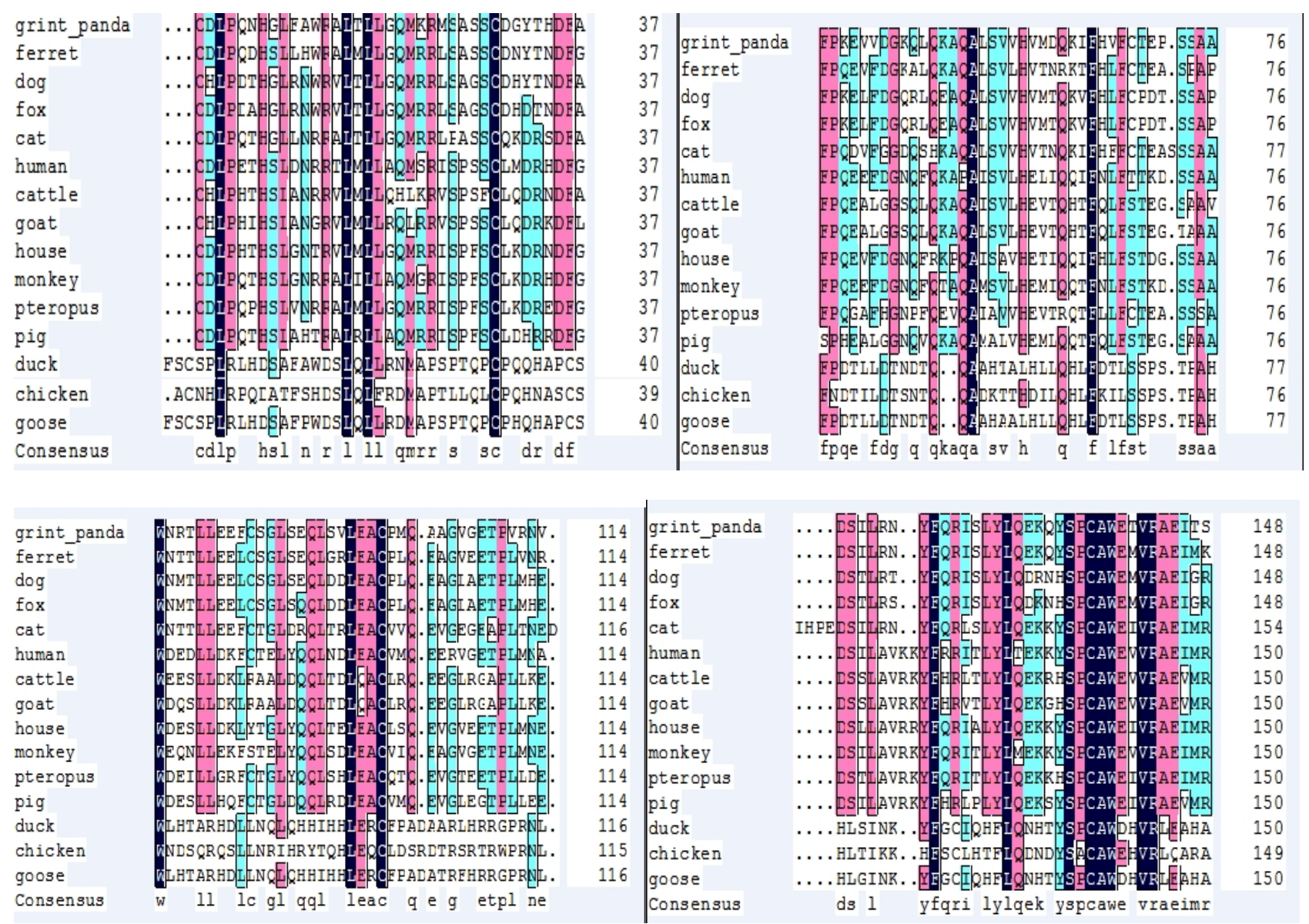

\begin{tabular}{|c|c|c|}
\hline grint_panda & 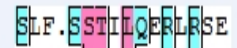 & 163 \\
\hline ferret & PLY.ASTVtLERRRSR & 163 \\
\hline $\operatorname{dog}$ & SEF. SSTILQREARRR & 163 \\
\hline fox & SEF. SSMILQRIRRR & 163 \\
\hline cat & SEYYSSTRARRRLRSE & 170 \\
\hline human & 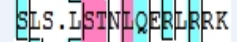 & 165 \\
\hline cattle & AES . SSTNRQESERRR & 165 \\
\hline goat & A|ES. SSMNLQEEEERK & 165 \\
\hline house & SES. SSTntECS.... & 161 \\
\hline monkey & 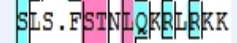 & 165 \\
\hline pteropus & SYS.LSTREKERSRSK & 165 \\
\hline pig & 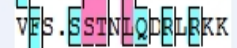 & 165 \\
\hline duck & CERRI HRLTRTMR... & 163 \\
\hline chicken & WELHI HNLTGNTRT. . & 163 \\
\hline goose & CERRI HRLTRAMR ... & 163 \\
\hline Consensus & sf sst lq $\mathrm{r} r$ & \\
\hline
\end{tabular}

On the basis of the alignment of the IFN- $\alpha$ sequences from 15 distinct genes, a phylogenetic tree of the interferon family had been constructed by using neighbor-joining method. The phylogenetic tree told us that the 15 interferons fell into two large groups. And there was an excellent relationship between the giant panda and ferret IFN $\alpha$. Further examination of the phylogenetic branch showed that the giant panda and ferret IFN $\alpha$ formed a monophyletic group distinct from
Figure 7. Amino acid sequence alignment of giant panda IFN- $\alpha$ from other species. The sequence alignments were performed using CLUSTALX.

dog and fox, confirming the similarity between giant panda and ferret IFN- $\alpha$ 's. The results also indicated that IFN-encoding regions of giant panda, ferret, dog and fox clustered together and evolved into a distinct phylogenetic lineage from that of mammals which evolved into another lineage. Phylogenetic analysis based on interferon sequences had also demonstrated that strains of the similar always clustered together. 


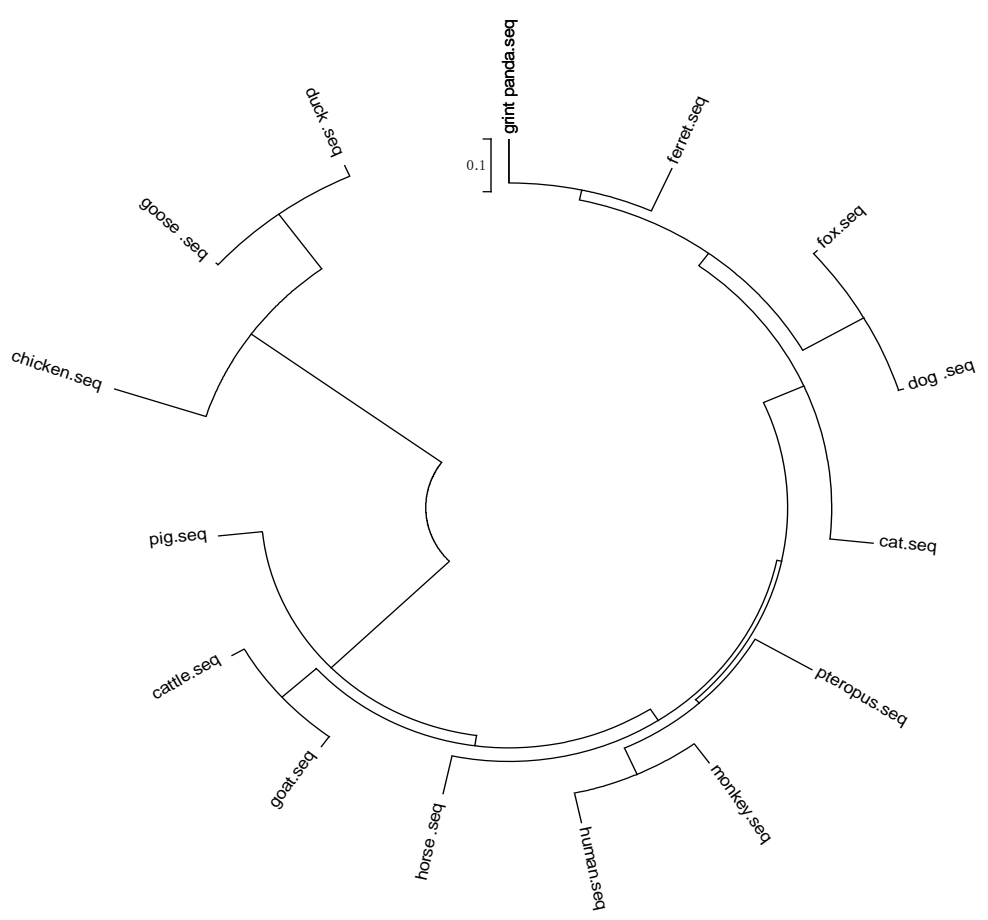

Figure 8. Phylogenetic relationships of IFN-a sequences from other species

\section{F. Rare condons analysis of the gpIFN- $\alpha$ gene}

Rare condons analysis showed that there were 15 rare condons $(9.15 \%)$ in the giant panda IFN- $\alpha$ gene by using codon usage database on line ( http://nihserver. mbi.ucla.edu/RACC/) . Five AGG codons and four AGA codons were the rare Arg condons. Two CTA codons were the rare Leu condons. Four CCC codons were the rare Pro condons. And also containing one consecutive rare codons AGACTAAGG.

\section{DISCUSSION}

The giant panda is one of the surviving ancient animals with high scientific value. It is considered as the fossil alived because of its represents of an important stage of evolution[8]. It has been reported that giant pandas are particularly susceptible to infectious disease and parasitic, which can result a $44 \%$ infection rate from pneumonia and tick-borne disease and a $66.67 \%$ mortality rate from ascariasis[9]. Therefore, it is very important to study the immune system of giant panda, especially immune function genes. In order to further understand the biological function of giant panda IFN- $\alpha$ at the molecular level and to extend the recombinant interferon-based applications in the precious animal, in this report, we describe the basic structure, function and characteristic of the $g p$ IFN- $\alpha$ through bioinformatics softwares and tools.

Signal peptides consist of short stretches of amino acids which, after protein delivery to the correct subcellular compartment, are frequently removed by specialized signal peptidases [10]. Only after removal of the signal peptide sequence, the precursor protein may be allowed to entry into the secretory pathway and become a normal function of the mature protein[11]. We plan to express the IFN-a gene in Ecoli expression system in the near future. So we clone the interferon alpha gene without single peptide. In the mature sequences there are six cysteine residues located in amino acid residues 1, 29, 69, 87,100 and 145. Four of these six cysteines (at positions $1,29,100$, and145) are conserved among most of the mammalian IFN-a proteins. The first residue of the mature peptide is cysteine residue which is important to disulfide bond formation and structure stabilization. Through the prediction of GeneScan, we find that the gpIFN- $\alpha$ gene is intronless, Similar with the other mammalian's IFN-a[12, 13]. A motif of 19 amino acids(YFQRISLYLQEKQYSPCAW) surrounding by 
Cys-145 is highly conserved among reference IFN- $\alpha$ sequences, which is a typical characteristic of Interferon alpha, beta and delta family, and may play an important role in the function of IFN-a.

Glycosylation is a complex, coor post-translational protein modification that serves to expand the diversity of the proteome [14]. It can influence the physical properties of the protein, including altered solubility, hydrophobicity, electrical charge and the protein thermal stability [15]. In the mature amino acid sequence of the gpIFN- $\alpha$, there are one potential N-glycosylation site, eight potential O-glycosylation sites, eleven potential phosphorylation sites and nine antigenic determinants. There are also twenty-one protein-protein interaction sites that are likely to be hot spots for protein interaction. That is, residues that are in the interface between two proteins and that are crucial for the stabilization of the complex. Analysis of the gpIFN- $\alpha$ protein through bioinformatics methods reveals that the protein may good immunogenicity and also undergochemical modifications like glycosylation and phosphorylation.

At the same time, there are 15 rare condons (9.15\%) in the giant panda IFN- $\alpha$ gene by using rare condons analysis. And one consecutive rare codons AGACTAAG $\mathrm{G}$ also were predicted. All the data initially indicated the excessive rare codons would affect gene's effective expression and the production of protein. Through analyzing the content of rare codons, it provided theoretical data not only for selecting the suitable host of expression bacterium, but also for improving the gene's expressing production with reforming codons[16] .

Phylogenetic trees infer the evolutionary relationships of species and patterns of gene duplications in multigene families[17]. In this study evolutionary relationship is analyzed by constructing a phylogenetic tree (shown in Fig. 9) based on the alignment of the IFN- $\alpha$ sequences from 15 distinct genes. The phylogenetic tree demonstrated that strains of the similar always clustered together.

In conclusion, bioinformatics are used to predict and analyze the molecular characteristics and evolution relationship of giant panda's IFN-a. All the consequences provide a basis data for futher research, We hope that the functional studies on the recombinant IFN-a in future could provide clues to new preventative and therapeutic treatments to the giant panda, which will be quite useful for the conservation of this species.

\section{ACKNOWLEDGMENT}

The authors are wish to thank Ya'an Research Base of Giant Panda Breeding for providing blood samples of the giant panda. Chengdong Wang contributed equally to this work and should be considered as first authors. Zhi-wen $\mathrm{Xu}$, Ling $\mathrm{Zhu}$ are the corresponding authors. Key Laboratory of Animal Disease and Human Health of Sichuan Province \& Animal Biotechnology Center, College of Veterinary Medicine of Sichuan Agricultural University, 46\# Xinkang Road, Yucheng district, Ya'an 625014, Sichuan province of China. Tel.: +86 835 2885477; fax: $+86 \quad 835 \quad 2885477$; E-mail address: abtcxzw@126.com. 


\section{REFERENCES}

[1] C. Samuel, "Antiviral actions of interferons," Clinical Microbiology Reviews, vol. 14, no. 4, pp. 778, 2001.

[2] H. Nguyen, J. Hiscott, and P. Pitha, "The growing family of interferon regulatory factors," Cytokine \& growth factor reviews, vol. 8, no. 4, pp. 293-312, 1997.

[3] M. Colonna, A. Krug, and M. Cella, "Interferon-producing cells: on the front line in immune responses against pathogens," Current opinion in immunology, vol. 14, no. 3, pp. 373-379, 2002.

[4] W. Stewart, and J. Vil ek, The interferon system: Springer-Verlag New York, 1979.

[5] P. Familletti, R. McCandliss, and S. Pestka, "Production of high levels of human leukocyte interferon from a continuous human myeloblast cell culture," Antimicrobial agents and chemotherapy, vol. 20, no. 1, pp. 5, 1981 .

[6] W. Feng, and G. Li, "The saving of giant panda," Published by Sichuan Science and Technology Publishing Company, pp. 113-129, 2000.

[7] X. Tan, Y. Tang, Y. Yang et al., "Gene cloning, sequencing, expression and biological activity of giant panda (Ailuropoda melanoleuca) interferon-[alpha]," Molecular immunology, vol. 44, no. 11, pp. 3061-3069, 2007.

[8] L. Xu, B. Zeng, R. Peng et al., "Molecular cloning and sequence analysis of the gene encoding interleukin- 6 of the giant panda (Ailuropoda melanoleuca)," Journal of Natural History, vol. 42, no. 39, pp. 2585-2591, 2008

[9] W. Feng, R. Wang, S. Zhong et al., "Analysis on the dead cause of the anatomical carcass of giant panda (Ailuropoda

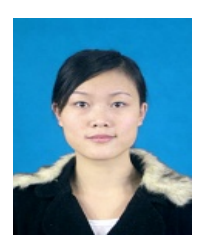

Yue Yi was born in sichuan, China, on March 1, 1987. Between the year 2005 and 2009, get bachelor's degree in Veterinary Medicine from Sichuan Agricultural University which is in Ya'an, Sichuan,and now she is a Post-graduated student sudy in Animal Biotechnology Center College of Veterinary Medicine of Sichuan Agricultural University for doctor degree of Prevention Veterinary Medicine. melanoleuca)," A study on breeding and diseases of the giant panda, pp. 244-248.

[10] H. Tjalsma, A. Bolhuis, J. Jongbloed et al., "Signal peptide-dependent protein transport in Bacillus subtilis: a genome-based survey of the secretome," Microbiology and Molecular Biology Reviews, vol. 64, no. 3, pp. 515, 2000.

[11] G. von Heijne, "The signal peptide," Journal of Membrane Biology, vol. 115, no. 3, pp. 195-201, 1990.

[12] R. Wonderling, T. Powell, S. Baldwin et al., "Cloning, expression, purification, and biological activity of five feline type I interferons," Veterinary immunology and immunopathology, vol. 89, no. 1-2, pp. 13-27, 2002.

[13] R. Devos, H. Cheroutre, Y. Taya et al., "Molecular cloning of human immune interferon cDNA and its expression in eukaryotic cells," Nucleic acids research, vol. 10, no. 8, pp. 2487, 1982.

[14] E. Weerapana, and B. Imperiali, "Asparagine-linked protein glycosylation: from eukaryotic to prokaryotic systems," Glycobiology, vol. 16, no. 6, pp. 91R, 2006.

[15] R. Huby, R. Dearman, and I. Kimber, "Why are some proteins allergens?," Toxicological Sciences, vol. 55, no. 2, pp. 235, 2000.

[16] S. Zhang, A. Cheng, M. Wang et al., "Molecular Cloning and Nucleotide Sequence Analysis of the Newly Identified UL53 Gene of Duck Enteritis Virus." pp. 1-6.

[17] S. Kumar, K. Tamura, and M. Nei, "MEGA3: integrated software for molecular evolutionary genetics analysis and sequence alignment," Briefings in bioinformatics, vol. 5, no. 2, pp. 150, 2004.

Zhiwen Xu was born in sichuan, China, on May 28, 1972. Received the B.S., M.S., and Ph.D. degrees from Sichuan Agricultural University of Prevention Veterinary Medicine in 1994, 1998, and 2003, respectively. Since 2010, He has been an Professor of Sichuan Agricultural University of Prevention Veterinary Medicine. His research interests include molecular biology, pathogenic microorganism. 